\title{
Development of Quantitative STEM for a Conventional S/TEM and Real-Time Current Calibration for Performing QSTEM with a Cold Field Emission Gun.
}

\author{
Stephen D. House ${ }^{1}$, Long Li ${ }^{1,2}$, C.T. Schamp ${ }^{3}$, Russell Henry ${ }^{3}$, Dong Su ${ }^{4}$, Eric Stach ${ }^{4}$, and Judith C. \\ Yang $^{1}$ \\ 1. Dept. of Chemical and Petroleum Engineering, University of Pittsburgh, Pittsburgh, PA (USA) \\ 2. (Current affiliation) RJ Lee Group, Monroeville, PA (USA) \\ 3. Hitachi High Technologies America, Inc., Dallas, TX (USA) \\ 4. Center for Functional Nanomaterials, Brookhaven National Laboratory, Upton, NY (USA)
}

The details of the size and shape of nanoparticle (NP) catalysts significantly impact their catalytic activity and effectiveness. Thus, the ability to characterize these materials at the relevant scales is critical to the rational design of improved catalysts. High-angle annular dark-field scanning TEM (HAADF-STEM) is particularly suited for the study of heterogeneous NP catalysts as it provides directly interpretable contrast primarily dependent upon the atom type and material thickness. Important information about the 3D structure, however, can be lost due to the 2D projection nature of TEM. Quantitative STEM (QSTEM) can recover atomic-scale 3D structural information from a single HAADF-STEM micrograph by taking advantage of the fact that with digital detectors, we are essentially counting electrons. Through careful calibration and measurement of the image and microscope, the contrast (scattered electron intensity) can be explicitly related back to the number of atoms involved in the scattering. This presentation discusses our developments on two different QSTEM approaches, one based on a conventional TEM/STEM and another on an aberration-corrected dedicated STEM.

The earliest QSTEM work performed demonstrated the feasibility of counting the number of atoms in ultra-small NP using sufficiently high collection angles ( $\geq 100 \mathrm{mrad})$ [1,2]. It was further shown that this method could also indirectly recover details of NP shape (e.g., spherical, hemispherical, or plate-like) [3]. Microscopes have advanced greatly in the intervening years, however, and the old VG STEMs used in these studies have all but disappeared. Here we present our adaptation of this method to enable its use on a conventional, non-aberration-corrected S/TEM, a JEOL JEM 2100F S/TEM with no special attachments or modifications to the microscope required. Two Au NP specimens, synthesized via UHV e-beam evaporation, were examined in this study: Au NP supported on an ultra-thin carbon (UC) film, and $\mathrm{Au} \mathrm{NP}$ deposited onto a $\gamma-\mathrm{Al}_{2} \mathrm{O}_{3}$ scaffold. The NPs were $<2 \mathrm{~nm}$ in size, typically $\sim 1 \mathrm{~nm}$. The necessary calibrations for QSTEM were accomplished by utilizing the free-lens control functionality of the JEM 2100F, allowing for the proper weighting and quantification of the intensity scattered from each NP. From these values, the scattering cross-section for each NP was calculated and the number of atoms determined, c.f., Figure 1.

More recently, the introduction of aberration-correctors enabled the development of atomic-resolution QSTEM, wherein by normalizing the intensities of an atomically resolved HAADF-STEM micrograph into units of fractional incident beam current, the number of atoms in each atomic column can be calculated through comparison with image simulations [4]. In combination with energy minimization computations, estimations of the particle's 3D morphology and atomic coordination can be reconstructed [5]. In this presentation we will discuss our development of a QSTEM technique on the aberration-corrected Hitachi HD2700-C STEM at Brookhaven National Laboratory. The cold field emission gun (CFEG) electron source of the microscope produces superior spatial coherence and energy 
spread than the more common Schottky FEG sources. The cost of these gains is a reduced stability of the emission current in a CFEG, which decays continuously (and non-linearly) and can vary even within an image. This renders existing methods for calibrating currents in QSTEM, which rely on a constant beam current, unsuitable for use with a CFEG. Our approach to overcome the instabilities of the CFEG is to measure the incident probe current in real-time concurrently with image acquisition. To do this, hardware has been installed to measure the signal from electrons impinging upon the objective aperture. By correlating this signal with the incident probe current, measured using a Faraday cup TEM stage, c.f., Figure 2, the aperture can act as a beam monitor, providing a measure of the instantaneous probe current without impeding imaging. By acquiring the beam monitor signal simultaneously with HAADF images, each pixel of the image can be calibrated individually using the incident beam intensity at the moment that pixel was acquired. In addition to enabling QSTEM to take advantage of CFEG sources, this method is also less impacted by the non-uniformities present in all imaging detectors [6].

\section{References:}

[1] MMJ Treacy and SB Rice, Journal of Microscopy 156 (1989), p. 211-234

[2] A Singhal, et al., Ultramicroscopy 67 (1997) p. 191-206

[3] JC Yang, et al., Materials Characterization 51 (2003), p. 101-107

[4] JM LeBeau, et al., Nano Letters 10 (2010), p. 4405-4408

[5] L Jones, et al., Nano Letters 14 (2014), p. 6336-6341

[6] This work was supported by DOE BES through grant DE FG02-03ER15476, and performed using the facilities at the Nanoscale Fabrication and Characterization Facility at the University of Pittsburgh and the Center for Functional Nanomaterials at Brookhaven National Laboratory, which is supported by DOE BES through contract DE-SC0012704.
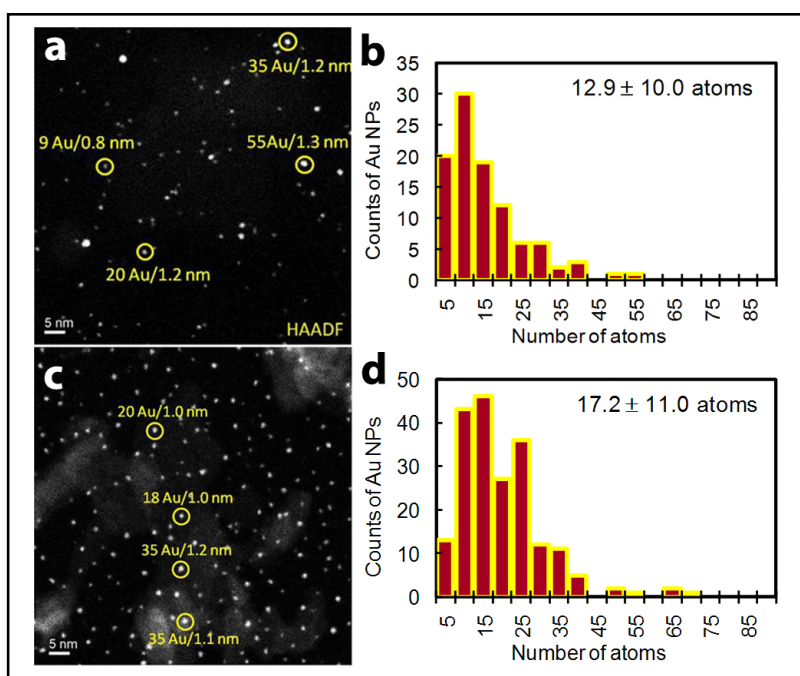

Figure 1. QSTEM measurements of $\mathrm{Au}$ NPs on $(a, b)$ UC film and $(c, d) \gamma-\mathrm{Al}_{2} \mathrm{O}_{3}$. (a) and (c) show HAADF-STEM micrographs with selected NPs labeled with their sizes. (b) and (d) plot histograms of cluster size.

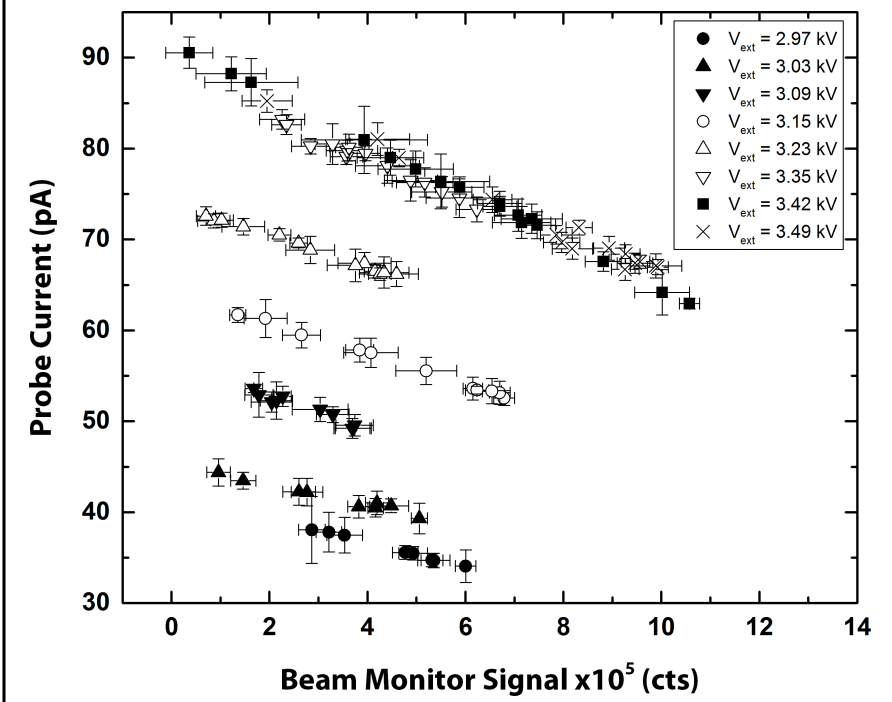

Figure 2. Probe current plotted versus beam monitor signal, showing a linear relation between the two, increasing with time/extraction voltage up to what appears to be a stable region. Error bars are the standard deviations. 